\title{
A Linear Approximation for the Excitation Energies of single and double analog states in the $f_{7 / 2}$ shell
}

\author{
Y. Durga Devi, Shadow Robinson and Larry Zamick \\ Department of Physics and Astronomy, Rutgers University, Piscataway \\ New Jersey 08854-8019, USA
}

\begin{abstract}
We find that the excitation energies of single analog states for odd-even nuclei in the $\mathrm{f}_{7 / 2}$ shell with $\mathrm{J}=\mathrm{j}=7 / 2^{-}$and the $\mathrm{J}=0^{+}$double analog states in the even-even nuclei are well described by the formulas $E^{*}(j, T+1)=b(T+X)$ and $E^{*}\left(0^{+}, T+2\right)=2 b(T+X+0.5)$, respectively, where $T=|N-Z| / 2$ is usually the ground state isospin. It is remarkable to note that the parameter $\mathrm{X}$ accounts for the departures from the symmetry energy based predictions.
\end{abstract}

Typeset using REVTEX 


\section{INTRODUCTION}

In a 1964 Technical Report, McCullen, Bayman and Zamick (M.B.Z) gave the wavefunctions and energy levels for nuclei in the $f_{7 / 2}$ shell [1]. In Table 1, we show their results for the excitation energies of single analog states in odd-even and even-odd nuclei, with angular momentum $\mathrm{J}=\mathrm{j}=7 / 2$, also shown are the calculated energies of double analog states in the even-even $\mathrm{Ti}$ isotopes.

It was noted by M.B.Z [1] that in some cases there was a two to one relation between the spectra of even-even nuclei and neighbouring odd A nuclei. For example the calculated $\mathrm{J}=0^{+}$spectra of ${ }^{44} \mathrm{Ti}$ were at twice the energies of the corresponding $\mathrm{J}=\mathrm{j}$ levels in ${ }^{43} \mathrm{Ti}$ (or

$\left.{ }^{43} \mathrm{Sc}\right)$. The same was true for the pairs $\left({ }^{48} \mathrm{Ti},{ }^{47} \mathrm{Sc}\right),\left({ }^{48} \mathrm{Ti},{ }^{49} \mathrm{Ti}\right)$, and $\left({ }^{52} \mathrm{Fe},{ }^{53} \mathrm{Fe}\right)$. That the two to one relation should hold was easily proved [2]. Since for these nuclei it was true for all levels (of the $\mathrm{j}^{n}$ configuration) which also includes the double and single analog states. It was noted by Zheng and Zamick [3] that the two to one relation holds quite well experimentally not only for the above mentioned pairs but for others as well. Zamick and Devi showed [2] that the two to one relation holds approximately for $\left({ }^{46} \mathrm{Ti},{ }^{45} \mathrm{Sc}\right)$ and the cross conjugate pair $\left({ }^{50} \mathrm{Cr},{ }^{51} \mathrm{Cr}\right)$. One gets an exact two to one relation here as well if one excludes seniority four states.

Besides the two to one relation there is the general question of the systematics of the excitation energies of the single analog states and double analog states. We will discuss this in the next section.

\section{EMPIRICAL FORMULA FOR ECITATION ENERGIES OF SINGLE AND DOUBLE ANALOG STATES IN THE $\mathbf{F}_{7 / 2}$ SHELL}

\section{A. Symmetry Energy Formula}

The symmetry energy in the semi-empirical mass formula is $-a_{S Y M}(Z-N)^{2} / A$ with $\mathrm{a}_{S Y M}$ equal to $-21.4 \mathrm{MeV}$.

Let us define $T=|N-Z| / 2$. For most nuclei $\mathrm{T}$ is the ground state isospin. With this formula the excitation energy of a single analog state is proprotional to $\left[(T+1)^{2}-T^{2}\right]=$ $2(T+1 / 2)$ whilst the energy of a double analog state is proportional to $\left[(T+2)^{2}-T^{2}\right]=$ $4(T+1)$. More precisely,

$$
E^{*}(S . A .)=-\frac{8 a_{S Y M}}{A}\left(T+\frac{1}{2}\right)
$$




$$
E^{*}(D . A .)=-\frac{16 a_{S Y M}}{A}(T+1)
$$

\section{B. The multipole interaction $a+b t(1) \cdot t(2)$}

The total potential energy will be $(b / 2) T \cdot T+$ constant

The S.A. energy will be $(b / 2)[(T+1)(T+2)-T(T+1)]=b(T+1)$

The D.A. energy will be $(b / 2)[(T+2)(T+3)-T(T+1)]=2 b\left(T+\frac{3}{2}\right)$

\section{A Linear Fit}

The previous simple models suggest that the energies are approximately linear in $\mathrm{T}$ (or $|N-Z|)$, we try

$$
\begin{gathered}
E(S . A .)=b(T+X) \\
E(D . A .)=2 b(T+X+1 / 2)
\end{gathered}
$$

In Table 1 we fit the above formulas to the calculated energies of single $\mathrm{j}$ shell calculations in which the spectrum of ${ }^{42} \mathrm{Sc}$ was used to determine the matrix elements $\left\langle\left(j^{2}\right)^{I}|V|\left(j^{2}\right)^{I}\right\rangle$, $j=f_{7 / 2}$ and $\mathrm{I}$ ranges from zero to seven. The matrix elements, in $\mathrm{MeV}$, are $0,0.6111$, $1.5863,1.4904,2.8153,1.5101,3.242$ and 0.6163 , respectively.

We find that we get a good fit with $\mathrm{b}=2.32 \mathrm{MeV}$ and $\mathrm{X}=1.30$. The formulas do not give an exact fit, but the results are nevertheless very good. For this linear fit there are several results which are independent of the values of the parameters b and X. For example, states with the same T should have the same S.A. excitation energies in the shell model calculation. However, the single j shell results are very close $4.142 \mathrm{MeV}$ and $4.112 \mathrm{MeV}$. Wherever the single $\mathrm{j}$ shell gives a two to one ratio for the energies of D.A. states as compared with S.A. states. So does the linear fit, irrespective of what $\mathrm{b}$ and $\mathrm{X}$ are.

The single $\mathrm{j}$ shell calculation does give two to one ratios of D.A. to S.A. for the pairs $\left({ }^{44} \mathrm{Ti},{ }^{43} \mathrm{Ti}\right),\left({ }^{44} \mathrm{Ti},{ }^{43} \mathrm{Sc}\right),\left({ }^{48} \mathrm{Ti},{ }^{49} \mathrm{Ti}\right)$ and $\left({ }^{48} \mathrm{Ti},{ }^{47} \mathrm{Sc}\right),\left({ }^{52} \mathrm{Fe},{ }^{53} \mathrm{Fe}\right)$ and so also do the linear formulas. But the linear formulas also give two to one ratios where the single $\mathrm{j}$ shell does not. These include $\left({ }^{46} \mathrm{Ti},{ }^{45} \mathrm{Sc}\right)$ and $\left({ }^{46} \mathrm{Ti},{ }^{47} \mathrm{Ti}\right)$. In the single $\mathrm{j}$ shell the excitation energies are $13.204 \mathrm{MeV}$ for ${ }^{46} \mathrm{Ti}$ and $6.590 \mathrm{MeV}$ for ${ }^{47} \mathrm{Ti}$ yielding a ratio 2.0036 .

The $\left({ }^{46} \mathrm{Ti},{ }^{45} \mathrm{Sc}\right)$ case was considered by Zamick and Devi [2]. They noted that if in the single $\mathrm{j}$ shell calculation one neglected seniority four admixtures then one would get a two to 
one relation because then the dimensions of the basis states would be the same - four (In the exact case they were 6 for ${ }^{46} \mathrm{Ti}$ and 7 for ${ }^{45} \mathrm{Sc}$ ). Indeed in the $4 \times 4$ diagonalization there will be a two to one relation for $\underline{\text { all }}$ the states, not just the analog state and as mentioned in ref. [2] even if one does not neglect seniority 4 states one can see by eyeball an approximate correction between the energies and wavefunctions of several of the states in the two nuclei.

However, the case $\left({ }^{46} \mathrm{Ti},{ }^{47} \mathrm{Ti}\right)$ is different. There are $17 \mathrm{~J}=\mathrm{j}$ basis states for ${ }^{47} \mathrm{Ti}$, but as previously mentioned, only, six for ${ }^{46} \mathrm{Ti}$. Nevertheless the D.A. analog excitation energy in the single $\mathrm{j}$ shell calculation is very close to twice that of the S.A. excitation energy in ${ }^{47} \mathrm{Ti}$. The actual ratio is 2.0036 .

Note also that the S.A. states in ${ }^{45} \mathrm{Sc}$ and ${ }^{47} \mathrm{Ti}$ have nearly the same excitation energies. Again the configurations look completely different. In ${ }^{45} \mathrm{Sc}$ we have one proton and four neutrons. The four neutrons could have seniority 0,2 or 4 . In ${ }^{47} \mathrm{Ti}$ we have 2 protons and three neutron holes. The only common thread between the two nuclei is that they have the same neutron excess $\mathrm{N}-\mathrm{Z}=3$. Indeed the linear approximation yields both the two to one relation between the D.A. in ${ }^{46} \mathrm{Ti}$ and the S.A. in ${ }^{47} \mathrm{Ti}$ and and the equality of the S.A. excitation energies of ${ }^{45} \mathrm{Sc}$ and ${ }^{47} \mathrm{Ti}$.

\section{THE EXPERIMENTAL SITUATION}

Previously Zamick and Zeng [3] made a comparison of single analog and double analog excitation energies with shell model calculations. While the main thrust of the present work is to discuss surprizing near degeneracies of SA and DA excitations in the single $\mathrm{j}$ shell model, a reexamination of the experimental situation would certainly be of interest and of value.

We will here make a fit to the D.A. excitations in the Titanium isotopes with our linear formula $\mathrm{E}^{*}(\mathrm{D} . \mathrm{A})=.2 \mathrm{~b}(\mathrm{~T}+\mathrm{X}+0.5)$. The results are as follows:

$\begin{array}{ccc}\text { Nucleus } & \mathrm{E}^{*}(\mathrm{D} . \mathrm{A} .)(\mathrm{MeV}) & 2 \mathrm{~b}(\mathrm{~T}+\mathrm{X}+0.5) \\ & \text { Experiment } & \mathrm{X}=2.00 \mathrm{~b}=1.94 \mathrm{MeV} \\ { }^{44} \mathrm{Ti} & 9.340 & 9.70 \\ { }^{46} \mathrm{Ti} & 14.153 & 13.58 \\ { }^{48} \mathrm{Ti} & 17.379 & 17.46\end{array}$

The values of $\mathrm{X}$ and $\mathrm{b}$ in Table. 1 are quite different $(\mathrm{X}=1.30, \mathrm{~b}=2.32)$. To get better agreement will require more extensive calculations involving configuration mixing, Coulomb interactions and A dependence of the parameters. Concerning the latter point it should be noted that in ${ }^{52} \mathrm{Fe}$, the value of $\mathrm{E}^{*}$ (D.A.) is $8.559 \mathrm{MeV}$. With fixed $\mathrm{b}, \mathrm{X}$ (or in a single $\mathrm{j}$ 
shell calculation with fixed two body matrix elements) this energy should be the same as that in ${ }^{44} \mathrm{Ti}$. We can parametrize the difference by assuming that $\mathrm{b}$ varies as $1 / \sqrt{A}$. This is different from the 1/A dependence of the previously mentioned symmetry energy in the semi-empirical mass formula.

In the previous references the experimental values for several S.A. and D.A. excitation energies were given. We will here give these and a few more, grouped according to the isospin. For half integer isospin we give the S.A. excitation energies while for integer isospin we give the D.A. excitation energies. The units are $\mathrm{MeV}$. We also list nuclei for which the energies are not known followed by a question mark. Hopefully this will stimulate experimental activity.

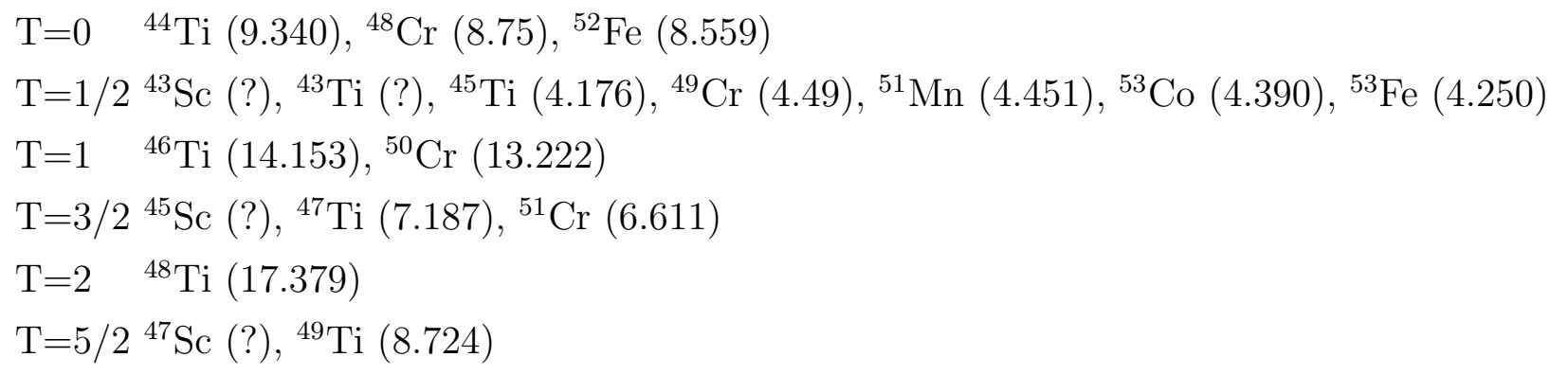

\section{CONCLUSIONS}

We find that we can give a very good but not perfect fit to a wide variety of single analog and double analog excitation energies by using the formulas $E^{*}(S . A)=.b(T+X)$ and $E^{*}(D . A)=.b\left(T+X+\frac{1}{2}\right)$ where $T=|N-Z| / 2$. The value of $\mathrm{X}$ is however different from that given by simple symmetry energy arguments. For fixed $\mathrm{b}$ the formulas predict that states with the same $\mathrm{T}$ will have the same excitation energies. In the single $\mathrm{j}$ shell calculation this is true in some special cases e.g. for cross conjugate pairs, but is not true in general. But even when not true the excitation energies are remarkably similar e.g. ${ }^{45} \mathrm{Sc}$, ${ }^{47} \mathrm{Ti}$. They differ by only $10 \mathrm{keV}$ in the single $\mathrm{j}$ shell calculation.

The above formulas give two to one ratios for the excitation energies of D.A. states of isospin $\mathrm{T}$ (integer) and S.A. states of isospin $T+\frac{1}{2}$. In some special cases the single $\mathrm{j}$ shell calculation also gives the result e.g. $\left({ }^{44} \mathrm{Ti},{ }^{43} \mathrm{Ti}\right)$. In other cases it does not but nevertheless the results are very close e.g. ${ }^{50} \mathrm{Cr},{ }^{51} \mathrm{Cr}$. The $\left({ }^{50} \mathrm{Cr},{ }^{51} \mathrm{Cr}\right)$ case can be explained by seniority truncation (i.e. neglecting seniority 4 admixtures) but the closeness of the single analog excitation in ${ }^{45} \mathrm{Sc}$ and ${ }^{47} \mathrm{Ti}$ cannot. In the former case the components of the wavefunctions look visually similar, but for ${ }^{45} \mathrm{Sc}$ and ${ }^{47} \mathrm{Ti}$ they look completely different.

A look at the experimental data shows that the grouping of excitation energies according 
to isospin $\mathrm{T}$ (or neutron excess $(\mathrm{N}-\mathrm{Z})$ ) is quite appropriate, although it would appear that the parameter $\mathrm{b}$ should have an $\mathrm{A}$ dependence. A rough analysis suggests that $\mathrm{b}$ is proportional to $1 / \sqrt{A}$.

In the future it will be of interest to see if we can get a better understanding of the parameter $\mathrm{X}$ i.e. why it differs from unity. Also the effect of coulomb energies and configuration mixing, which was partly dealt with in ref. [3] could be extended.

\section{Acknowledgements}

This work was supported by the Department of Energy Grant No.DE-FG02-95ER40940. 


\section{REFERENCES}

[1] J.D. McCullen, B.F. Bayman and L. Zamick, Phys. Rev. 134B (1964); Technical Report NYO-9891.

[2] L. Zamick and Y.D. Devi, Phys. Rev. C (1999) to appear in print.

[3] L. Zamick and D.C. Zheng, Phys. Rev. C46 (1992) 815. 


\section{TABLES}

TABLE I. The Excitation Energies of Single Analog ( $\mathrm{J}=\mathrm{j})$ states (S.A.) and Double Analog $\left(\mathrm{J}=0^{+}\right)$States (D.A.). A comparison is made of single $\mathrm{j}$ shell calculations using the spectrum of ${ }^{42} \mathrm{Sc}$ as input and linear fits.

\begin{tabular}{cccc}
\hline \hline Single Analog & Single $\mathrm{j}$ & $\mathrm{b}(\mathrm{T}+\mathrm{X})^{\mathrm{a}}$ & Formula \\
& $\mathrm{MeV}$ & $\mathrm{MeV}$ & \\
${ }^{43} \mathrm{Ti}\left({ }^{53} \mathrm{Co}\right){ }^{\mathrm{b}}$ & 4.142 & 4.176 & $\mathrm{~b}(0.5+\mathrm{X})$ \\
${ }^{45} \mathrm{Ti}$ & 4.112 & 4.176 & $\mathrm{~b}(0.5+\mathrm{X})$ \\
${ }^{45} \mathrm{Sc}\left({ }^{51} \mathrm{Cr}\right)$ & 6.601 & 6.496 & $\mathrm{~b}(1.5+\mathrm{X})$ \\
${ }^{47} \mathrm{Ti}\left({ }^{49} \mathrm{~V}\right)$ & 6.590 & 6.496 & $\mathrm{~b}(1.5+\mathrm{X})$ \\
${ }^{49} \mathrm{Ti}\left({ }^{47} \mathrm{Sc}\right)$ & 8.829 & 8.816 & $\mathrm{~b}(2.5+\mathrm{X})$ \\
${ }^{\mathrm{Double} \mathrm{Analog}}$ & & & \\
${ }^{44} \mathrm{Ti}\left({ }^{52} \mathrm{Fe}\right)$ & $2 \mathrm{~b}(\mathrm{~T}+\mathrm{X}+1 / 2)^{\mathrm{a}}$ & $2 \mathrm{~b}(0.5+\mathrm{X})$ \\
${ }^{48} \mathrm{Cr}$ & 8.352 & $2 \mathrm{~b}(0.5+\mathrm{X})$ \\
${ }^{46} \mathrm{Ti}\left({ }^{50} \mathrm{Cr}\right)$ & 8.284 & 8.352 & $2 \mathrm{~b}(1.5+\mathrm{X})$ \\
${ }^{48} \mathrm{Ti}$ & 8.000 & 12.992 & $2 \mathrm{~b}(2.5+\mathrm{X})$ \\
\hline
\end{tabular}

${ }^{\mathrm{a}} b=2.32 \mathrm{MeV} \quad X=1.30$

$T=|N-Z| / 2$

${ }^{\mathrm{b}}$ Also the Mirror Nuclei ${ }^{43} \mathrm{Sc}\left({ }^{53} \mathrm{Fe}\right)$ 